(Aus der dritten Medizinischen Klinik der Universität Berlin. [Direktor: Geheimrat Prof. Dr. Goldscheider.])

\title{
Beitrag zur Klinik der Relaxatio diaphragmatica.
}

\author{
Ton
}

\author{
E. Herzfeld und W. Unverricht. \\ Mit 2 Textabbildungen. \\ (Eingegangen am 26. Oktober 1921.)
}

Pathologische Veränderungen am Zwerchfell sind klinisch besonders in den letzten zwei Jahrzehnten näher studiert worden. Das hatte seinen Grund darin, daß erst mit der Vervollkommnung des Röntgenverfahrens uns Änderungen in der Form und Bewegung des Diaphragma sichtbar gemacht werden konnten. So vermochten wir mit Hilfe der Röntgenstrahlen das seltene Vorkommen einer Zwerchfellhernie schon am Lebenden zu erkennen. Differentialdiagnostisch gegenüber letzterer macht der einseitige Zwerchfellhochstand, die Relaxatio diaphragmatica, Schwierigkeiten. Die Zahl der bisher beschriebenen Fälle von Relaxatio diaphragmatica ist relativ klein. Bergmann hat die bis zum Jahre 1913 bekanntgewordenen Beobachtungen zusammengestellt und einer kritischen Besprechung unterzogen. Nachträglich sind noch eine Reihe weiterer Fälle beschrieben worden. Thre differentialdiagnostische Abgrenzung gegenüber der Hernie ist oft unsicher, charakteristisch dafür sind einige Fälle, die einmal als Hernie, das andere Mal als Relaxatio in der Literatur niedergelegt wurden. Wir hatten Gelegenheit, auf der Klinik zwei Fälle von Relaxatio diaphragmatica zu beobachten, deren Mitteilung uns wegen der Seltenheit der Beobachtung geboten erscheint.

1. Fall Gertrud M., 20 Jahre alt. Der Vater ist an Lungentuberkulose gestorben. Pat. selbst will bis zum Herbst 1918 stets gesund gewesen sein. Damals erkrankte sie an Grippe und klagt seit dieser Zeit über Stiche in der linken Brustseite und Atemnot, die besonders nach körperlicher Anstrengung und während der Nacht auftreten. Da angeblich in letzter Zeit die Beschwerden zunahmen, wurde sie in die Klinik aufgenommen (Herzfeld).

Es handelte sich um eine untersetzte, aber ziemlich kräftig gebaute Pat. in leidlichem Ernährungszustand, mit mäBig durchbluteten Schleimhänten. Niemals Temperaturerhöhung. Atmung regelmäßig, nicht beschleunigt. Der Brustkorb von normalem Bau, die unteren Lungenränder rechts vorn in der Parasternallinie am unteren Rande der 6. Rippe, in der Scapularlinie in Höhe des 10. Brustwirbels, gut verschieblich; links hinten in der Scapularlinie befindet sich der untere Lungen- 
rand in Höhe des 7. und 8. Brustwirbels, er ist bei der Atmung nur sehr gering verschieblich. In den linken seitlichen Partien geringe Schallverkürzung, sonst perkutorisch und auseultatorisch kein krankhafter Befund. Das Röntgenbild zeigt - abgesehen von beiderseits verstärkten Hilusschatten - die linke Zwerchfellhälfte als dünnen hochstehenden gewölbten Schattenstrang, wesentlich höher als rechts, mit seiner Kuppe etwa in Höhe der 4. Rippe vorn. Bei der Durchleuchtung bewegt sich dieser Schatten bei der Einatmung zunächst etwas nach oben und dann unbedeutend nach unten, während er sich bei der Ausatmung gering nach aufwärts bewegt.

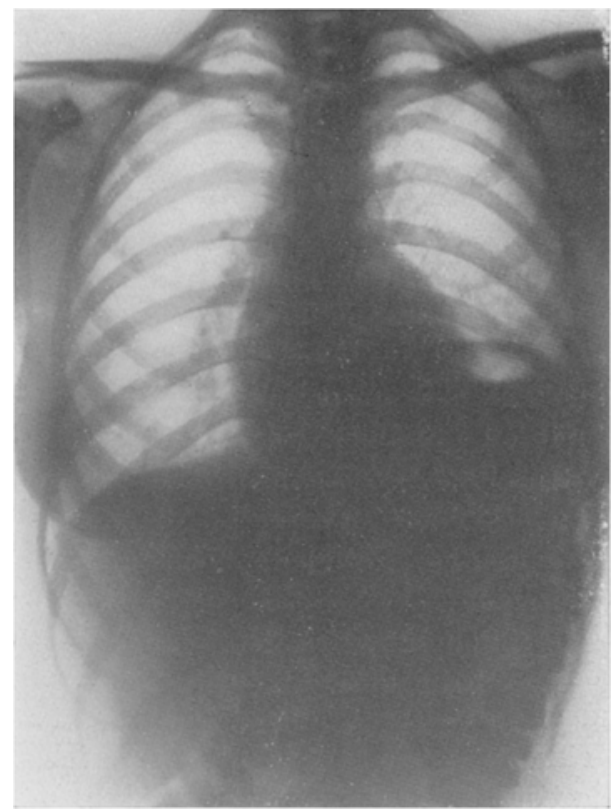

Abb. 1. Fall 1 (Gertrid M.). Eine faradische Reizung des Phrenicus am Halse ergab keine sichtbarenAusschlägedesZwerchfells. Nach Einnahmedes Bariumkontrastbreies zeigt es sich, daß der Brei sich unterhalb des bogenförmigen Schattenstranges ansammelt. Beim Neigen der Pat. auf die linke Seite blieb die Form des Schattens unverändert, während sich der Spiegel des Breies unterhalb des Stranges horizontal einstellte. Gleichgültig für die Form des Schattenstranges wax es auch; ob der Magen leer oder gefuillt war. Das abgebildete Röntgenbild (siehe Abb. 1) war noch bei Füllung mit Kontrastbrei aufgenommen, wodurch der Gesamteindruck vielleicht nicht ganz so deutlich erscheinen dürfte, wie bei den mehrfachen von uns vorgenommenen Durchleuchtungen. Die relative Herzgrenze befindet sich links innerhalb der Mamillarlinie, während dieselbe nach rechts den rechten Sternalrand um $1 \mathrm{~cm}$ überragt. Es sei noch erwähnt, daß während der Krankenhausbeobachtung bei der auBerdem recht nervösen Pat. Anfälle von Atemnot nicht zur Beobachtung kamen.

Noch ausgesprochener als in dem eben geschilderten Falle finden wir das Bild der Relaxatio bei der anderen Patientin.

2. Fall Geo., 34 Jahre alt, außer Kinderkrankheiten keinerlei Erkrankung. 1917 eine linksseitige Pleuritis exsudativa, bei der zweimal eine Punktion gemacht sein soll. Pat. wurde damals als geheilt aus dem Krankenhaus entlassen, litt jedoch noch längere Zeit unter Schmerzen in der linken Seite beim Atemholen. Seit Februar 1919 zunehmende Mattigkeit, Fusten und wenig Auswurf. Pat. wurde dem einen von uns (Unverricht) zwecks Pneumothorax-Behandlung überwiesen.

Befund: Grazile Frau in mäßigem Frnährungszustand von leidendem Aussehen. Atmung leicht beschleunigt, 20-24 Atemzüge in der Minute. Rechte untere Lungengrenze hinten am 10. Brustwirbeldorn gut verschieblich. Links am Dornfortsatz des linken Brustwirbeldorns unverschieblich. Rechts hinten oben bis 
Hilus, vom bis 3. I.C.R. Dämpfung, vesicobronehiales Atmen nit feinen, an der Spina auch mittleren Rasselgeräuschen. Herz: Spitzensto B verbreitert innerhalb der Mamillarlinie. Grenzen: Links ein Querfinger innerhalb der Mamillarlinie, rechts iberschreitet sie etwa um ein halb Zentimeter den rechten Sternalrand. Sputum: Etwa $30 \mathrm{cem}$ täglich. Tbc-Bacillen +. Elastische Fasern negativ. Röntgenbefund: Marmorierung der oberen Hälfte des rechten Lungenfeldes, links Zwerchfellhälfte ragt als feiner gewölbter Schattenstrang weit in das linke Lungenfeld hinein und reicht mit ihrer Kuppe bis zur vorderen 3. Rippe. Bei gewöhnlicher Atmung sind keine Exkursionen sichtbar. Dagegen. macht es eine kleine paradoxe Bewegung beim Bittorfschen Vexsuch. Eine faradische Reizung des Phrenicus am. Fialse bewirkt eine geringere Bewegung der Zwerchfellhälfte nach abwärts. Nach Einnehmen eines Kontrastbreies sieht man eine deutliche Ansammlung der Mahlzeit unterhalb der Bogenlinie. Bei Lagerung auf die linke Seite bleibt die Bogenlinie, auch selbst bei leichter Beckenhochlágerung erhalten. Unterhalb der Bogenlinie bewegt sich der Spiegel des Kontrastbreies deutlich je nach der Lagerung und stellt sich immer horizontal ein. Bei Ausuibung eines Diuckes auf die gefiullte Magenblase behalt die Bogenlinie in we Begrenzung. Auf́blähung des Magens mit Kohlensäure ändert ebensowenig wie Auspumpen der Luft mittels eines Magenschlauches die Form der bogenförmigen Schattenlinie.

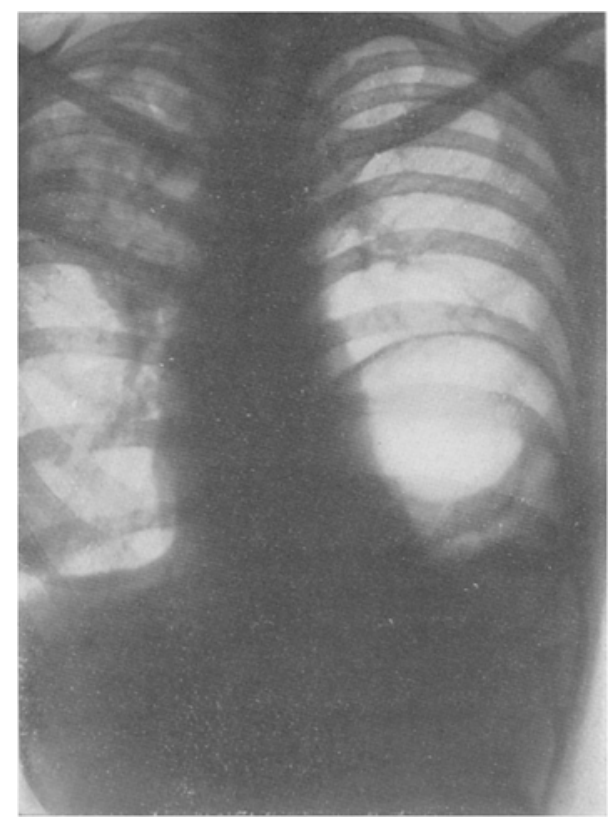

Abb. 2. Fall 2 (Geo.),

In beiden Fällen handelt es sich also um eine linksseitige Relaxatio diaphragmatica. Die rechtsseitige ist noch viel seltener und von Giässner und Eppingex beobachtet worden. Die Diagnose unserer beiden Fälle darf - soweit klinisch möglich - als gesichert gelten. Allerdings haben wir die bereits von Neumann vorgeschlagene und von Schlecht und Wels ausgeführte Anlegung eines Pneumoperitoneum zwecks differentialdiagnostischer Abgrenzung gegenüber der Fernia diaphragmatica nicht angewendet, denn dieser Eingriff ist unserer Meinung nach nur indiziert, falls eine Entscheidung auf andere Weise nicht möglich ist. Früher wurde angenommen, daß eine parajoxe Bewegung der Zwerchfellhälfte für Hernie spreche. Doch hatte Ass. mann behauptet, daB theoretisch eher das Gegenteil anzunehmen wäre, eine Ansicht, die in letzter Zeit durch eine Beobachtung von 
Leendertz bestätigt wurde. Auch der eine unserer Fälle zeigte bei Bittorfschem Versuch paradoxe Bewegung der betr. Zwerchfellhälfte. Der eine von uns (Unverricht) hat durch klinische und experimentelle Beobachtung die Annahme sehr wahrscheinlich gemacht, daß für das Zustandekommen der paradoxen Bewegung der Tonusverlust maßgebend sei. Ein relaxiertes, wie mehrfach durch Autopsie festgestellt, stets muskulär geschädigtes $Z$ werchfell hat natürlich keinen Tonus mehr, auch wenn die Nervenleitung noch intakt ist. Die paradoxe Bewegung bei dem einen unserer Fälle spräche also für Relaxatio. Das Fehlen bei anderen beschriebenen Fällen dürfte seine Ursache in adhäsiven pleuritischen Prozessen haben. Die Reizung des Phrenicus brachte uns in differentialdiagnostischer Hinsicht nicht weiter. Ein Fall reagierte, der andere nicht. Eine doppelte Bogenkontur, wie sie Assmann als sicheres Merkmal der Relaxatio besehreibt, konnten wir niemals wahrnehmen. Entscheidend für die Diagnose unserer beiden Fälle halten wir das unveränderte Bestehenbleiben des Bogenschattens bei Neigen und nach Lagerung auf die linke Seite bei mit Brei gefülltem Magen und nach Druck auf die Magenblase. Diese Tatsache zwingt uns zu dem Schluß, daß die Bogenlinie als Zwerchfell anzusprechen ist. In demselben Sinne ist das Fehlen jeglichen Einflusses von die Form der Magenblase verändernden Eingriffen auf die Kontur der Bogenlinie zu verwerten. Die Füllung des Magens mit Kontrastbrei, die Aufblähung des Magens mit Kohlensäure und Auspumpung der Luft sowie von außen ausgeübter Druck brachten keine Änderung des Bildes. Ätiologisch ist ein Angeborensein nicht auszuschließen, wenn auch in beiden Fällen das Überstehen einer Pleuritis mit sekundärer Schädigung der Zwerchfellmuskulatur als ursächliches Moment wahrscheinlich ist. 